\title{
La isla de los olvidados: los docentes y el modelo educativo por competencias en la educación superior tecnológica
}

\author{
The island of the forgotten: the teachers and the educational model by competences in
} technological higher education A ilha dos esquecidos: os docentes e o modelo educativo por competências na educação superior tecnológica

DOI: http://dx.doi.org/10.21803\%2Fpenamer.11.21.530

Guillermo Isaac González Rodríguez https://orcid.org/0000-0002-4584-1773

\section{¿Cómo citar este artículo?}

González, G. (2018). La isla de los olvidados: los docentes y el modelo educativo por competencias en la educación superior tecnológica. Pensamiento Americano, 11(21), 136-152

DOI: http://dx.doi.org/10.21803\%2Fpenamer.11.21.530

\section{Resumen}

En el presente artículo se abordan los puntos que ha afrontado en su implementación el "Modelo Educativo para el Siglo XXI: Formación y Desarrollo de Competencias Profesionales" en la educación superior tecnológica. Dicho modelo surge en el 2013 para dar un cambio a la educación que imparten los Institutos Tecnológico-descentralizados (ITS) en México. El trabajo es de tipo descriptivo y se utilizó un estudio de caso, en un tecnológico de Jalisco para conocer la percepción de los docentes sobre el mismo. Se aplicó una entrevista semi-estructurada a un grupo de 20 docentes de diferentes departamentos del ITS en Zapopan, Jalisco. La tarea fue analizar la interpretación que tienen los docentes sobre el proceso de implementación del modelo educativo y sus consecuencias. Se parte de la idea de conocer ¿cuál es la interpretación y percepción que tienen los docentes sobre la implementación del modelo educativo por competencias? Los resultados se plasman en un cuadro interpretativo sobre los cambios realizados y los objetivos alcanzados. Como conclusiones se puede observar que los cambios no han sido claros y los docentes llevan a cabo el modelo, pero sin conocer lo que implica el cambio curricular y las nuevas estrategias de enseñanza-aprendizaje.

Palabras clave: Modelo educativo, retos docentes, interpretación docente, competencias.

\begin{abstract}
The present article addresses the points that have been faced in its implementation of the "Educational Model for the XXI Century: Training and Development of Professional Competencies" in higher technological education. This model emerged in 2013 to give a change to the education provided by the decentralized Technological Institutes (ITS) in Mexico. The work is of a descriptive type and a case study was used, in a technological of Jalisco to know the perception of teachers about it. A semi-structured interview was applied to a group of 20 teachers from different ITS departments in Zapopan, Jalisco. The task was to analyze the interpretation that teachers have about the process of implementation of the educational model and its consequences. It starts from the idea of knowing what is the interpretation and perception that teachers have about the implementation of the educational model by competencies? The results are reflected in an interpretative table about the changes made and the objectives achieved. As conclusions, it can be observed that the changes have not been clear and the teachers carry out the model, but without knowing what the curricular change implies and the new teaching-learning strategies.
\end{abstract}

Keywords: educational model, teaching challenges, teacher interpretation, competences. 


\section{Resumo}

$\mathrm{Na}$ presente article se abordam os pontos os quais se confrontou em sua implementação o "Modelo Educativo para o século XXI: Formação e Desenvolvimento de Competências profissionais", na educação superior tecnológica. Dito modelo surge em 2013 para realizar uma mudança na educação que efetuam os Institutos Tecnológicos Descentralizados (ITS) no México. $O$ trabalho é de tipo descritivo e se utilizou um estudo de caso, em um tecnológico de Jalisco para conhecer a percepção dos professores sobre o mesmo.

Se aplicou uma entrevista semi-estruturada a um grupo de 20 professores de diferentes departamentos do ITS em Zapopan, Jalisco. A tarefa foi analisar a interpretação que os docentes possuem sobre o processo de implementação do modelo educativo e suas consequências. Se parte da ideia de conhecer qualéa interpretação e a percepção que têm os docentes sobre a implementação do modelo educativo por competências? Os resultados se plasmam em um quadro interpretativo sobre as mudanças realizadas e os objetivos alcançados. Como conclusões se pode observar que as mudanças não foram claras e os docentes terminam o modelo proposto, mas sem conhecer o que implica a mudança curricular e as novas estratégias de ensino-aprendizagem.

Palavras-chave: Modelo educativo, retos docentes, interpretación docente, competencias.

\section{Perfil}

Guillermo Isaac González Rodríguez

Maestro en Gestión y

Política de la Educación

Superior.

Tecnológico Superior de Zapopan. México.

Correo electrónico: guillermo.gonzalez@itszapopan.edu.mx

Doctorante en Gestión de la Educación Superior, Universidad de Guadalajara.

Docenteinvestigador en el Instituto 


\section{Introducción}

La

a manera más radical de imponer un cambio es realizarlo de manera impositipor medio de represión, castigos y normas se trataba de cambiar la manera de actuar de los individuos. En esa isla se presentaron formas alternativas de educación que modificaba la manera de ser, aprender y pensar acorde a las necesidades sociales y contextuales. Esto generaba una base distintiva que homologaba las relaciones e interacción de los miembros. La metáfora de esta isla de los olvidados nos lleva al análisis de un modelo educativo en el espacio de la educación superior tecnológica de México. Dicho modelo es parte de una serie de cambios que se han desarrollado en la totalidad del sistema educativo superior tecnológico con la intención de fomentar el desarrollo de competencias profesionales en los alumnos. Cabe resaltar que la educación superior tecnológica es sólo un pequeño eslabón de las instituciones que conforman todo el campo universitario en México, dado que el sistema está conformado por 12 tipos distintos de instituciones que prestan educación superior. Una derivación de ellas son los Institutos Tecnológicos que aglomeran a 266 instituciones distribuidas en todo el territorio de México y que se distinguen por ser federales (IT) y descentralizados (ITS) dependiendo de su configuración organizacional y la estructura financiera y jerárquica. Antes de describir el factor que abarca a la educación tecnológica debemos de contextualizar el medio ambiente donde se desenvuelven.

A partir de la vorágine espaciotemporal desarrollada desde la década de los ochenta y consolidada en los noventa, se han realizado cambios en varios espacios sociales, económicos y culturales en distintos países. Los espacios como las universidades son ahora una fuente de complejidad que integra a distintos personajes, procesos y conceptos modernos. Aspectos como la calidad, la evaluación, la acreditación, el financiamiento, el emprendimiento, la competitividad, la internacionalización, la cooperación y el curriculum se convirtieron en ejes centrales de las acciones que se toman dentro de las universidades. Es por eso que se observó la necesidad de generar un espacio que le facilitara a la Educación Superior (ES) crear un campo homogéneo de intercambio y cooperación para generar nuevas experiencias educativas.

Lo anterior sirve de guía para posicionar los espacios educativos creados a raíz de esta serie de intercambios para la implementación de nuevas estrategias de enseñanza-aprendizaje. Es por ello que la ES de México en la época de la modernización(noventa) buscó alternativas apegadas a las tendencias mundiales y atendió las recomendaciones hechas por instancias internacionales (OCDE, BM, UNESCO) sobre la mejora educativa. Una de estas opciones fue la diferenciación educativa, que llevó a la creación de nuevas instituciones como los Institutos Tecnológicos Descentralizados (ITS), las Universidades Tecnológicas (UT), las Universidades Politécnicas (UP), entre otras, que dieron entrada a nuevos modelos educativos. En este trabajo se hace un acercamiento a la implementación del Modelo Educativo Siglo XXI (MSXXI) en los diversos tecnológicos.

El caso de Jalisco es particular pues en el año 2016 se creó una instancia que congrega a los trece ITS bajo el auspicio de una entidad denominada Instituto Tecnológico Mario Molina (TecMM). Esta es una primera aproximación al fenómeno de estudio y sirve de base para la creación de un instrumento que nos permita aplicarlo en los trece tecnológicos que existen en el estado de Jalisco. Por tanto, sólo se presenta la experiencia en el Instituto Tecnológico Mario Molina campus Zapopan (TecMMZ) como un inicio de la investigación 
que trata de rescatar la percepción docente sobre el modelo educativo por competencias. EI MSXXI lleva implícito un cambio desde tres perspectivas; la académica, la curricular y la organizacional. Es un cambio significativo en lo que respecta a la forma en que se presta educación tecnológica, pues cambian los planes, los programas y las planeaciones de clase. Resulta interesante comprender cómo está funcionando el MSXXI respecto al modelo anterior y desde esta perspectiva se realizó este primer acercamiento.

Mediante una metodología cualitativa y el uso del método de estudio de caso, efectuamos entrevistas semi-estructuradas a una muestra de 20 docentes de tiempo completo del TecMMZ. De las entrevistas surgieron categorías de análisis que utilizamos como base para el desarrollo de la interpretación y los retos que tienen los docentes ante este cambio. Las categorías van desde la práctica docente, los retos a los que se enfrentan, las ventajas y desventajas del modelo, las carencias personales, proceso de implementación. El trabajo está dividido en dos apartados y una conclusión, en donde se explica en qué consiste el MSXXI; para después hacer una extrapolación sobre lo que son las competencias profesionales y la labor docente; posteriormente se describe el escenario de implementación; para terminar, se presentan los que arrojaron las entrevistas.

\section{El modelo educativo como parte de una propuesta.}

En primer lugar, debemos de definir lo que es un modelo y como primera instancia nos encontramos con que Magalys Ruíz (2001) describe al modelo desde su concepción más básica como; "una abstracción de la realidad (política), pero que requiere una operacionalización (implementación) que promueva la acción desde tres planos; teórico, empírico y formalizado (Ruíz, 2001, p. 15)" Por tal motivo el modelo está enmarcado por el espacio subjetivo de cómo interpreta una realidad que le presentan (políticas), a una realidad de quien lo modela (gestores educativos) o pone en ejecución (docentes). El caso de formular un modelo distinto en un contexto específico confiere realizar un diagnóstico situacional que nos arroje problemáticas específicas, fundamentos teóricos, estrategias y metodologías que nos permitan formular las directrices de este (Escudero \& Martínez, 2017).

Con este supuesto, el modelo educativo debe ir encaminado a desarrollar las herramientas con las que los docentes, directivos y académicos lleven a cabo su práctica al momento de buscar la mejora en los procesos y en la formación de los estudiantes. Es por eso que la buena instrumentación del modelo como una política de cambio establecerá la forma en que realice la transmisión del conocimiento dentro del aula de clase y se reflejará en los estudiantes al momento de ponerlo en práctica en su ámbito profesional (Cordero, Contreras, Fernandez, \& Hernández, 2016). Los distintos modelos en la educación superior y en particular en las universidades, tiene varios referentes importantes que pueden tomarse como base para la creación de una nueva disciplina, de nuevos procesos académicos y de inserción en la investigación (Stan, 2017).

Por un lado, tenemos lo planteado por Peter Senge (2002) en su escuela que aprende, donde propone un manual de la quinta disciplina, pensado para educadores, padres de familia y todos los interesados en la educación. En este manual, se rescata la labor que deben de tener los interesados en la educación, planteando que las escuelas deben de aprender, y de donde parte la premisa de las disciplinas necesarias para el aprendizaje cooperativo. Tales disciplinas son: dominio personal, visión compartida, modelos mentales, aprendizaje en equipo y pensar en sistemas (Senge, 2002), que generen un ambiente participativo y de 
reciprocidad en la generación de conocimiento y convivencia generadora de aprendizaje.

Por otro lado, Paúl Wildman (2003), plantea siete temas emergentes para las universidades futuras, en donde destaca la forma para establecer un modelo pertinente. Según lo establece, se deben tomar en cuenta varios aspectos como lo son: la economía del conocimiento, la globalización, la capacidad que tenga la comunidad donde se aplicará, el desarrollo de pedagogías alternativas, la visión de una economía post-mercado, las posibilidades de futuros fragmentados y neo-renacimiento de costumbres y tradiciones (pp. 131135). El análisis de los retos a enfrentar puede definir el rumbo que tomará el modelo y establecerá las pautas con las que formulen las bases teórico-metodológicas que les darán vida. Con estos puntos, se puede establecer una propuesta sobre la universidad polifónica, que rescata la labor de la universidad como una institución que mediatiza la transmisión del conocimiento y genera un modelo adecuado al contexto (Wildman, 2003).

Al igual que la educación debe tomar lo mejor de varios modelos para lograr la participación e inclusión social, el docente debe de redimensionar su papel de mediador, precursor, divulgador, juez y parte de los procesos de aprendizaje y enseñanza. Lo que se busca con esto es que los alumnos y la sociedad sean partícipes de los mismos, y no sólo espectadores y receptores de la información (Ortega, 2017). Para ello, deben afrontar los retos que plantea la creación de sociedades del conocimiento, las cuales requieren de nuevos modelos de adaptación que no creen rezago ni exclusión social, y a su vez, desarrollen participación colectiva. Todo modelo educativo se debe de establecer desde cuatro ejes fundamentales de búsqueda y encuentro administrativo y académico. Estos ejes son:
- El análisis del binomio educación-sociedad

- La concepción curricular

- Las concepciones de enseñanza-aprendizaje

- La cultura organizacional que se rige en medio para la influencia de la concepción modélica (Ruíz, 2001, p.56)

Para que esto sea posible es imperioso implementar un modelo que permita reflexionar y crear profesionales en lo que hacen (Díaz Barriga, 1997), personas capaces de solucionar problemáticas, de concebir pensamientos coherentes, capaces de reflexión histórica, de implementar proyectos en beneficio de la sociedad, fomentar el desarrollo y capaces de tener un razonamiento crítico del entorno sociopolítico-económico al cual pertenecen (Garzón, 2016).

Por tanto, un modelo educativo en el plano de la implementación y aplicación por parte de los docentes, debe de ser o establecerse como una representación conceptual-simbólica de la realidad a la cual estará enmarcado (García-Varcárcel, 2002), también es visto como una representación de la realidad con la finalidad de delimitar algunas de sus dimensiones (Escudero, 1981), Los modelos educativos que buscan el desarrollo de competencias requieren de ciertas características para que puedan resultar funcionales en el contexto donde se implementen (García, Junyent, \& Fonolleda, 2017). Se puede establecer que un modelo basado en las competencias debe de movilizar conocimiento, tener una teoría solida que lo respalde, definir una metodología y estrategias de acción, centrar la intención den el aprendizaje y llevar una estructura bien definida sobre sus objetivos (García, 2011). Siendo ellos un modo de generar capacidades distintas en los sistemas donde se produce conocimiento, los modelos tienen diversas formas de interpretarse cuando surgen de la intencionalidad y no de la colectividad (Stan, Koniotaki, \& Religa, 2018). De ello deriva que 
en los sistemas educativos exista una distinción sobre los procesos que se llevan a cabo con el modelo y las estrategias con las cuales se pone a andar en un contexto determinado (Zapata, 2015).

\section{Implicaciones del modelo por competen- cias en la educación superior tecnológica}

En México, el sector educativo es diverso ya que el origen de las instituciones educativas depende en gran medida del contexto y tipo de institución o nivel que abarque. El sistema educativo se presta en seis niveles: preescolar (de dos a tres años), primaria (seis años), secundaria (tres años), preparatoria (3 años), licenciatura (cuatro años) y posgrado (la duración es depende el grado-maestría, especialidad o doctorado). El nivel licenciatura y posgrado tienen subdivisiones acordes al tipo de educación que se preste. En nivel licenciatura existen instituciones que se encargan de impartir educación con base tecnológica que son conocidos como los Institutos Tecnológicos y que como ya lo mencionamos, se dividen en dos tipos los IT y los ITS. Cada uno de ellos con algunas modalidades y carreras distintas, pero bajo un mismo modelo educativo (Villagrán., Jasso., Aldaba, \& Rodríguez, 2017).

Este tipo de instituciones cobraron fuerza a partir de los años cincuenta, donde la industrialización del país tuvo un auge significativo y era necesario que la educación prepara a los profesionales en el área de ingeniería. Tiempo después, en la década de los noventa, nacen los ITS que se diferencian de los IT porque sus recursos provienen en una parte proporcional del gobierno federal y otra del estatal. Esta clase de instituciones han tenido un gran impulso, pues a la fecha a lo largo de México existen 134 divididos en todos los estados. Con ellas se busca crear una opción distinta para la formación de estudiantes bajo un enfoque que pueda solventar las carencias y necesidades de las regiones con programas y planes ad hoc a los distintos contextos donde se ofertan (TecNM, 2015).

Actualmente dichas instituciones son reestructuradas desde la parte académica, organizacional y normativa mediante tres etapas esenciales que llevan un proceso iniciado desde el año 2006. La primera etapa es la reestructuración de los programas y planes bajo el enfoque de competencias profesionales realizado en el año de 2010. En esta etapa se realizó una reestructuración curricular para todos los planes en busca del cambio en el enfoque. Todos los programas tuvieron cambios y ahora la labor de los docentes para realizar su planeación e implementar estrategias implica un conocimiento distinto de sus funciones. La segunda etapa fue el diseño y puesta en marcha del MSXXI en el año 2013. Este modelo llego a suplir el antiguo modelo que estuvo en funciones 7 años y que era el primer indicio de una educación integral. Y la tercera etapa fue la creación del Tecnológico Nacional de México (TecNM) en el año 2014 como entidad rectora que aglomera a la totalidad de los tecnológicos en México. Estas etapas han establecido un cambio en el sistema educativo de los IT en diversas perspectivas, pero no han sido un foco de atención para los estudios realizados sobre procesos de cambio en México (González, 2014).

Para esta investigación se tomó sólo un caso de estudio para ejemplificar la manera en que han repercutido estos cambios efectuados en la labor de los docentes. Desde la implementación del modelo se establecieron objetivos específicos para que los docentes adoptaran el modelo, pero no se definieron de manera clara las estrategias con las que se pretendía lograr. Lo que se tomó en consideración al momento de realizarlo, fue el entorno macro puesto que se pensaba en un modelo para la educación básica que pudiera establecer una reforma en este nivel educativo, por lo que 
presentó el cambio en el nivel medio superior y tecnológico también. Ello conlleva que las directrices apuntaran a un nuevo sistema de gestionar este tipo de instituciones para conseguir una reestructuración total de mismo y unificar el sistema (Villagrán, et. al 2017).

Cabe resaltar que la educación tecnológica abre la posibilidad para comprender los diversos contextos donde se presta este tipo de educación, pues en algunas regiones, es la opción más viable, o incluso la única que tienen las personas para estudiar ES. He ahí la importancia de comprender el modelo desde su desarrollo hasta su puesta en marcha en el contexto de los programas y planes de la malla curricular. Este modelo está orientado a establecer pautas de cambio en los procesos educativos, organizacionales y axiológicos para centrar sus objetivos en la formación de profesionales competentes (SNEST, 2013).

En el presente caso, se decidió realizar el trabajo en el TecMMZ pues es el que concentra al mayor número de alumnos, docentes y administrativos de los campus de ITS que se encuentran en Jalisco. Dicha institución inició sus operaciones con la finalidad de impulsar el desarrollo en la Zona Metropolitana de Guadalajara en materia de innovación, ciencia y tecnología desde el año 1999. En la actualidad cuenta con 7 carreras, 6 de las cuales son ingenierías (Electrónica, Electromecánica, Sistemas, Civil, Gestión Empresarial e Industrial), una licenciatura (Gastronomía), y un posgrado en Sistemas Computacionales. Cuenta con una planta docente de 216 profesores, 78 de los cuales cuentan con posgrado, 16 de ellos con grado de doctor, 3 en especialidades y los demás se encuentran en el grado de maestría terminada o en curso. Las carreras que cuentan con más profesores son la de Industrial (36), Electromecánica (31) y Sistemas (29) (ITSZapopan, 2016).
En esta institución como en todas las demás se comenzó a implementar el MSXXI con el desconocimiento completo de los docentes sobre lo que debían hacer o la manera en que deberían actuar dentro y fuera del aula. Las funciones que cambiaron tenían implicaciones diversas que repercutían con la planeación de clase, el diseño de sus estrategias y la búsqueda del desarrollo de competencias en losalumnos. El diseño de la malla curricular iba en función de lograr generar estas competencias, ya que la estructura de los planes va en consecuencia de ello y están definidos según el contexto. Por su parte, los programas están diseñados para que el docente pueda realizar su planeación basado en todos los aspectos de las secuencias didácticas y las estrategias de acción, por lo que es necesario adquirir las competencias y habilidades docentes que les hagan poner en marcha el modelo y cumplir con ello el objetivo que se tiene.

Según lo específica en la parte de justificación, el modelo educativo está estructurado para lograr la formación y desarro-llo de competencias profesionales necesarias en la actualidad para el auge del capital humano (SNEST, 2013). Dicho modelo es abordado desde tres puntos transversales, los cuales se articulan para complementar los procesos de enseñanza-aprendizaje en los alumnos. Los ejes van desde la parte axiológica, la académica y la organizacional. Fue diseñado y puesto en marcha antes de la creación del TecNM, por lo que los objetivos y líneas estrategias siguen el mismo curso, pero ahora bajo otra instancia rectora. Para que pudiera comprenderse el proceso, se creó un manual de operaciones, con el que se sientan las bases para establecer la evaluación, las salidas terminales, la movilidad estudiantil, las residencias profesionales, tutorías y las funciones de las distintas academias de los que estudian en algunos de los tecnológicos del país (TecNM, 2015). 
Las nuevas reglas dan forma a la implementación del modelo educativo desde distintas arenas académicas y administrativas, pero a su vez, generan en los encargados directos de la implementación, los docentes, diferentes factores que permean su accionar, dentro y fuera del aula de clase (González, 2014). En este caso, el modelo trata de impulsar el ejercicio y fomento de las competencias profesionales en el ámbito tecnológico en rela־ción al conocimiento, las formas de pensar, y la capacidad para actuar de los estudiantes (SEP, 2013). La intención de desarrollar competencias profesionales va encaminada a proporcionar a los individuos las herramientas para participar de forma pertinente en su entorno y que haya una repercusión que impacte socialmente (SEP, 2013).
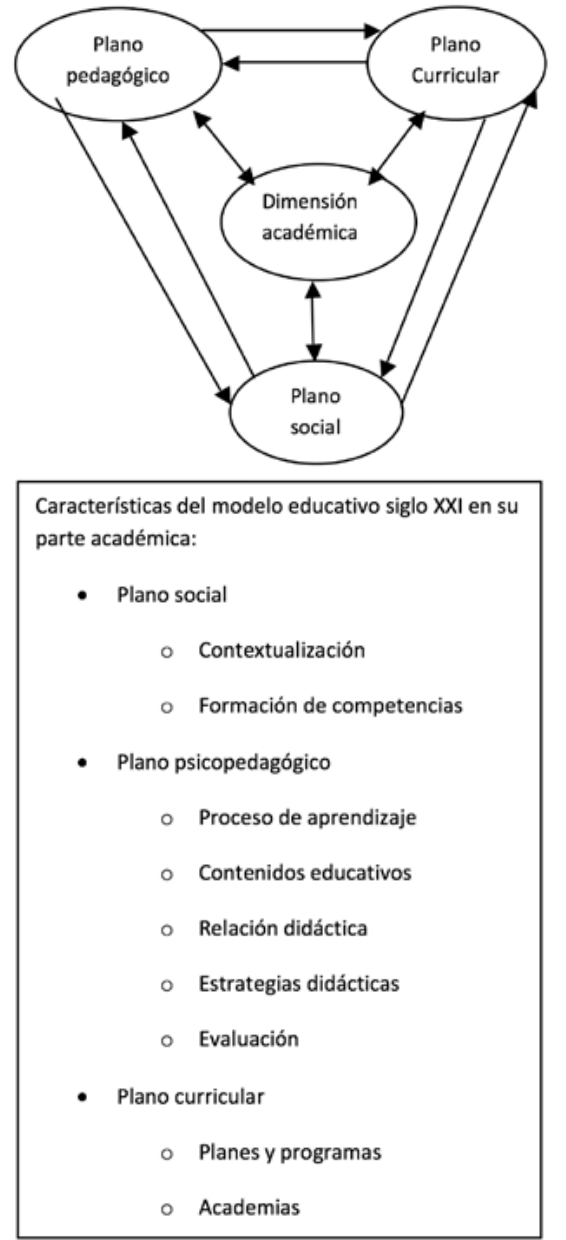

Diagrama 1: Modelo educativo siglo XXI por competencias Elaboración propia tomada de: Modelo educativo siglo XXI (SEP, 2013)
El modelo está dividido en tres ejes centrales que le dan forma y estructura a todo el proceso educativo: la parte axiológica establece los valores que se busca transmitir y el origen del modelo; la académica conlleva todos los planes, programas, curriculum y formación de los docentes; y la organizacional que genera las líneas por las cuales el modelo será implementado (SNEST, 2013). El modelo tiene la intención de mejorar la calidad educativa mediante la adaptación de la curricula a las necesidades actuales que la industria, la sociedad y el contexto global tienen (SNEST, 2013). Al hacer una síntesis de la dinámica con la cual se desarrolló el documento y al tomar como base lo que se pretende alcanzar con la implementación de este, se obtienen ciertos puntos de articulación y objetivos cuyas principales líneas rectoras se enfocan en:

- Incrementar el número de programas académicos reconocidos por su buena calidad y de procesos certificados.

- Elevar la calidad de los servicios educativos en las instituciones del SNIT.

- Fomentar la atención de temas urgentes y emergentes para mejorar las con-diciones de vida de todos los mexicanos (SNEST, 2013).

En este caso, los IT e ITS ofrecen programas educativos acordes con la demanda de las diversas regiones del país y sus necesidades (TecNM, 2017). Se trata entonces de que las competencias profesionales se desarrollen en los estudiantes mediante una configura ción intelectual que integre, en su estructura y funcionamiento, una forma diferente de pensamiento. En el siguiente apartado se comienza a describir el desempeño de las competencias en el caso desde la función de los docentes.

\section{La interpretación docente sobre la imple- mentación del modelo por competencias.}

Para comprender mejor el fenómeno de la 
implementación de un modelo educativo, se decidió realizar un estudio que reflejara la percepción e interpretación que tienen los docentes sobre los nuevos procesos. A partir del desarrollo de estrategias específicas de acción nos dimos a la tarea de analizar esta percepción acorde a las tareas y funciones que tienen los docentes en el aula. La intención de ello fue rescatar de viva voz a los que están directamente involucrados en la implementación del modelo pero que no son tomados en cuenta en el proceso de formulación del mismo. Según lo establece la teoría, los principales factores para que un modelo pueda desarrollarse de buena manera, es que todos los involucrados tengan completo conocimiento sobre el mismo para que así los objetivos se cumplan.

Fue por ello que la elección metodológica realizada, está enfocada en analizar la interpretación de los docentes, específicamente desde su labor en la creación, desarrollo e implementación del modelo por competencias. Se eligió el método de estudio de caso donde se estableció una población de análisis con una muestra de docentes de las distintas academias y con diferentes perfiles para realizar la entrevista piloto. De este grupo se seleccionaron a 20 como muestra del total del universo, a quienes se les realizó una entrevista semi-estructurada derivada del análisis de los objetivos, preguntas de investigación, ejes temáticos y variables de análisis con un instrumento que consta de 12 preguntas para conocer lo que interpretan los docentes sobre su actuar, hacer y evaluar en el modelo.

Para el análisis e interpretación se utilizaron categorías que mediante el software Atlas Ti se esquematizaron para poder realizar el cuadro de sugerencias. Para el presente caso, se fueron entrelazando lo mencionado por los docentes con la confrontación que generan entre ellos por la interpretación que hacen del modelo y su implementación. Lo que se obtuvo es que varios docentes difieren de la forma de trabajo bajo este modelo y lo consideran muy dócil al momento de evaluar, pues suponen que no tiene, o implica mayor labor en la creación de las competencias. Lo esencial viene desde la interpretación que hacen de las competencias y del papel que juegan para crearlas. Se considera que las competencias profesionales son una configura ción intelectual que integra en su estructura y funcionamiento una forma diferente de pensamiento, con base en la reflexión y critica del entorno (García Fraile, Tobón, \& López, 2008). En tanto, la profesión es tomada como una práctica social que se caracteriza por contar con una serie de actividades, las cuales se desa $\neg$ rrollan con base en un conjunto de conocimientos especializados, capacidades intelectuales, aptitudinales y actitudinales que requieren del compromiso personal y la responsa-bilidad por parte de quien la ejerce (Díaz-Barriga \& Hernández, 2002).

Del análisis de las entrevistas y la interpretación de las categorías surgió el siguiente diagrama donde se pueden ver las líneas por las que se describen las entrevistas que se presentan en el siguiente apartado.

En el diagrama se pueden apreciar las categorías que arrojó el análisis en el software y con el cual nos basamos para hacer la construcción de este apartado. Se parte de la interpretación docente sobre el modelo y las implicaciones que ha traído a su labor como docentes. Otro apartado es la definición que tienen sobre el modelo y los recursos que utilizan para ponerlo en práctica. Otra más data sobre las estrategias que adoptaron para cambiar su visión respecto al modelo. Se sigue con el grado de participación que tienen sobre el mismo y los problemas que encontraron o encuentran a la hora de implementarlo. Finalmente se tratan de observar las funciones que tiene el mode- 
lo en la creación de competencias y lo que se necesita para mejorar y adaptarlo a sus prácticas cotidianas, ya que, los docentes también necesitan desarrollar las competencias para implementar el modelo.

En este caso, el MSXXI trata de impulsar el ejercicio y fomento de las competencias profesionales en rela-ción a factores como el conocimiento, las formas de pensar, y la capacidad para actuar (SEP, 2013). La intención de desarrollar competencias va encaminada a proporcionar a los individuos las herramientas para participar de forma pertinente en su entorno. En relación con esto, Lasnier (2000) define las competencias como: "Saber hacer complejo resultado de la integración, movilización y adecuación de capacidades y habilidades (pueden ser de orden cognitivo, afecti- vo, psicomotor o sociales) y de conocimientos (conocimientos declarativos) utilizados eficazmente en situaciones que tengan un carácter común (situaciones similares, no generalizable a cualquier situación) (Lasnier, 2000, p. 13)."

Por otro lado, Gonczi y Athanasou (1996) precisan que "la competencia se define como una compleja estructura de atributos necesarios para el desempeño de situaciones específicas. Es una combinación de atributos (conocimiento, actitudes, valores y habilidades) y las tareas que se tienen que desempeñar en determinadas situaciones (Gonczi \& Athanasou, 1996, p. 27)." Desde esta perspectiva, las competencias deben de crearse en un ambiente donde el modelo pueda ser aplicado y a su vez fomente el cambio en los involucrados (Díaz-Barriga \& Hernández, 2003). Algu-

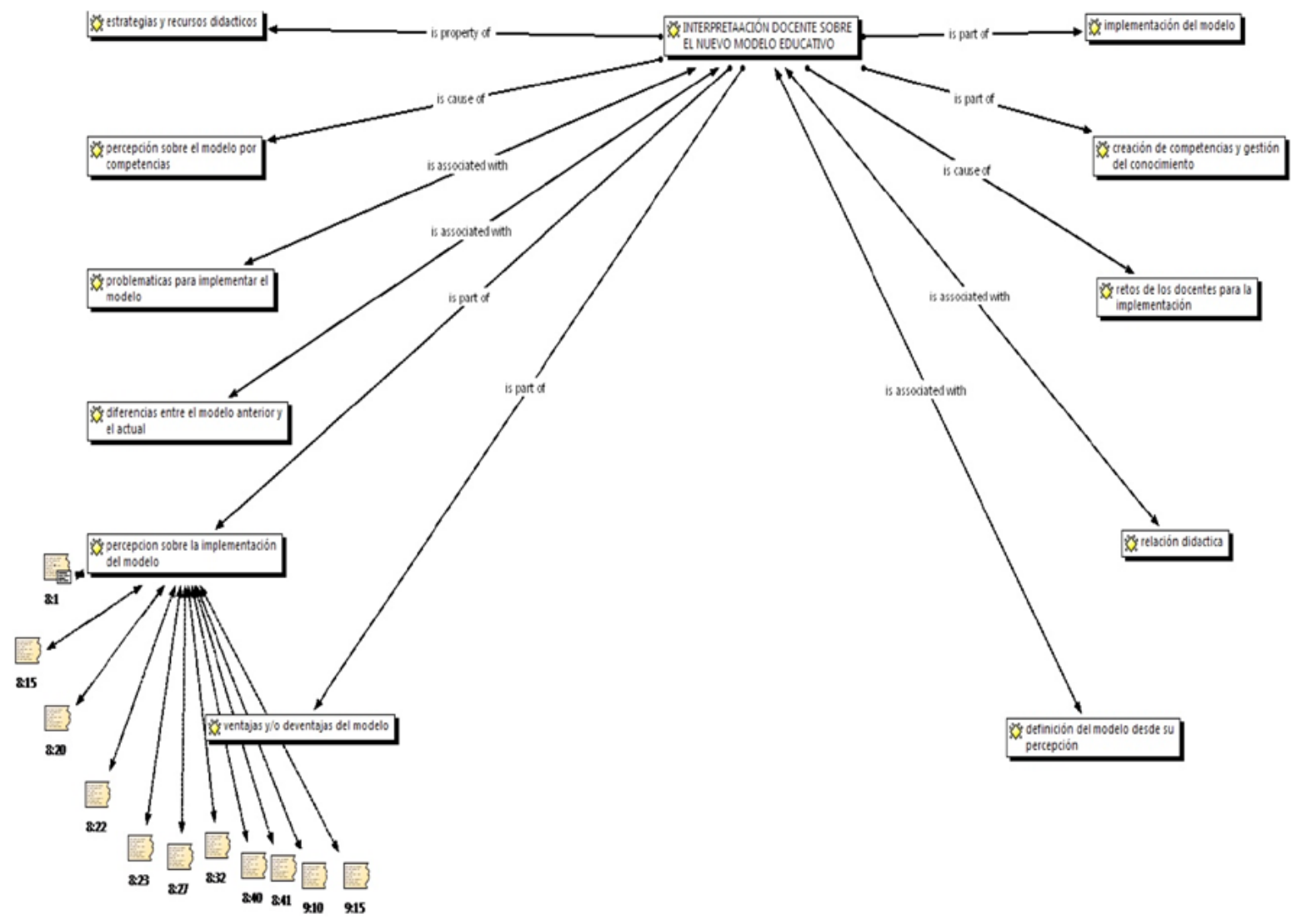


nos profesores difieren de la opinión de que el modelo por competencias tiene para la creación de las mismas competencias, y creen que no es aplicable en el entorno que ellos tienen. Consideran que tiene algunas ventajas y desventajas, pero que necesitan mayor conocimiento del mismo, pues, en las entrevistas varios de ellos explicaron que no conocen el modelo y que no han recibido capacitación para aplicarlo.

Las ventajas es que está un poquito más actualizado, pues al haberse reformado tiene temas más actuales, se están actualizando, la ventaja es que tiene muchas actividades ya desarrolladas, muchas sugerencias de otras actividades y pues, una desventaja es el desconocimiento del modelo al principio, todavía existen lagunas en cuanto a la forma de aplicarlo (D5).

Los docentes a los que se les aplicó la entrevista generaron opiniones en pro y en contra del MSXXI pues especificaban que el modelo generó un cambio curricular en los programas y planes. Consecuencia de esto fue que el trabajo y la dedicación a la planeación aumentó en gran medida, y el desarrollo de competencias es más complicado. Desde esta panorámica, pudimos detectar cómo repercute en su práctica, su hacer, su actuar y su pensar.

Ha generado un problema por el tiempo, porque si yo manejara el sistema por competencias tal como lo indica el modelo es más tiempo el que tengo que dedicarle yo a revisiones, a etapas de ejercicios que ellos tienen que hacer. Esto me implica que trabaje más y que el tiempo no me alcance al momento de evaluar (D17).

Tomamos algunas de las interpretaciones de los docentes para conocer si realmente el modelo puede tener una ventaja para su puesta en marcha. Lo ven como algo que llevará tiempo en adaptarse, y que necesita paciencia en su aplicación, y sobre todo esfuerzo y dedicación por parte de docentes, directivos y alumnos. A pesar de que lleva cerca de 4 años desde que se implementó, no han notado un avance significativo o un cambio sustancial en el desarrollo de competencias en los alumnos. Los docentes perciben el modelo con ventajas desde la parte curricular, pues los planes están actualizados. Sin embargo, perciben el modelo descontextualizado y ajeno a las situaciones que ellos viven dentro del aula de clase.

Es muy amigable en cuanto al proceso de evaluación, pero sí implica más tiempo, conocer bien el modelo y que las instituciones realmente entiendan lo que es el modelo por competencias, porque a veces se le deja la carga más bien, la carga del conocimiento al maestro...y la institución no se da cuenta cómo debe funcionar el modelo, yo siento que el modelo está un poco, o un mucho, fuera de contexto... (D7)

La mayoría ven muchas trabas en el modelo educativo, algunos en el aumento del trabajo, otros en el poco conocimiento sobre el mismo, o sobre cómo llevarlo a la práctica. Desde la perspectiva del desarrollo curricular, cuando se formula un modelo nuevo, deben de estar involucrados todos los participantes para que no haya discrepancias, "El que hace los modelos educativos, yo creo que no tiene la menor idea de lo que pasa en el aula. Entonces, no pueden ellos decir, esto es lo mejor cuando nunca han estado acá (D1)" Los docentes hablan desde su experiencia sobre el proceso que se lleva a cabo para ejecutar este modelo y los resultados que se esperan obtener mediante la evaluación de los resultados en la educación tecnológica.

Yo me imagino que en un año o dos quizá ya podamos ver los resultados, cuando ya empiecen a egresar los alumnos con este 
modelo, y que ya las mismas empresas se vayan dando cuenta que nuestros alumnos van adquiriendo más habilidades (D3)

Como resultado de las entrevistas, notamos que los docentes utilizan el modelo porque es parte su labor pero tienen varias lagunas sobre los significados reales que tiene desde su concepción axiológica y curricular, "Yo siento que si mejoraría, si yo también tratara de entender cómo trabajar con él, pero, pues yo tampoco he tratado de entenderlo, no he podido entender el cómo llevar las misma materias a este sistema por competencias (D20)" Al momento de realizar las entrevistas, notamos esta percepción era recurrente en la mayoría de los docentes, pues fuera de la grabación, nos revelaban su sentir real, el cual no difería en muchas ocasiones de lo que contestaban en las preguntas. Es claro que el cambio los tomó por sorpresa y no lo han asimilado como parte significativa de su desempeño y labor.

Los docentes, desde su interpretación, consideran que no fueron tomados en cuenta para la implementación del modelo; tampoco fueron capacitados para ejecutarlo y nunca fueron informados de su puesta en marcha. Por tal motivo, los tomó por sorpresa y ha causado una serie de repercusiones en su práctica, así como en los tiempos y la manera de trabajar

Para mí si es un poquito más trabajo, me refiero a (no porque sea más chamba), que de repente es más complicado que entren los alumnos al modelo, porque, uno le puede entrar como puede, como sabe, o como quiere. Entonces, es un reto el decir: aquí estamos por competencias, pero no sabemos cómo eran las competencias anteriormente. A lo mejor lo estamos haciendo mal también, al decir; jesto es competencia! Y total, que tratamos de dar lo mejor que se pueda sin en ocasiones saber el resultado (D16)
Por tanto, sienten una ausencia de fondo al tratar de asimilar lo que el modelo establece, pues desconocen lo que el modelo para la educación tecnológica busca alcanzar. Lo tratan de aplicar bajo el entendimiento que ellos tienen sobre la forma de crear competencias y lo que ellos pueden llevar a cabo para tratar de formarlas, dado que como se mencionó, los recursos de aplicación no son los suficientes para que se sientan en plena libertad de establecer los objetivos del modelo. Esto es la base de la comparación del modelo anterior que dejaba la creación de conocimiento a la manera en que el docente mostraba su conocimiento práctico

En lo particular, en el modelo anterior a mí se me hacía en cierta forma más fácil en cuanto a la evaluación. Porque únicamente estábamos esperando un número, el resultado de las prácticas, entrego o no entrego, y bueno, en este modelo por competencias estamos evaluando... es una evaluación más integral porque estamos viendo no nada más la cuestión técnica, sino específicamente la cuestión de un conocimiento desarrollado (D14).

Todo esto genera en el docente una cierta incertidumbre en cuanto al modelo y su implementación, ya que podría parecer que la forma como se dice que se ejecuta tal como está establecido y lo que en realidad se hace, son dos cosas totalmente diferentes, tanto de comprender, como de aplicar y más aún cuando lo que se pretende es cambiar el esquema y la visión hacía la inserción de una nueva política reguladora y generadora de capital humano aprovechable.

Por tanto, algunos docentes del estudio conocen los principios básicos del modelo (o de la política) pero no sienten que sea viable por su aplicación en el ámbito al cual pertenecen. Lo que genera que opten por llevar a la práctica 
lo que han realizado desde hace varios años

Yo considero que el modelo y la forma con la que quieren que trabajemos, genera una problemática por el tipo de materias que cada quien imparte; algunas son más prácticas que teóricas. Si fueran materias un poco más teóricas entoncesyo digo que si sería un buen, una buena metodología. Poder utilizar y diseñar técnicas... técnicas que usualmente pues se habla de ellas, pero yo en lo personal no las practico (D8)

Existe también la opinión de que el modelo necesita, aparte de los docentes, que los alumnos también sean conscientes del proceso que se debe de llevar a cabo y de la responsabilidad que implica tanto para el docente como actor de la implantación, como para el alumno que es el receptor de la implantación, y finalmente es el mismo alumno en quien se verá reflejado el trabajo que llevo a cabo el docente durante el curso.

Es por eso que la responsabilidad recae en ambos actores que intercambian dinámicas de trabajo e información para la obtención de un producto. En este espacio, los docentes tienen un propósito específico; desarrollar en los alumnos la competencia de aprender a aprender para alcanzar el propósito formativo del proceso educativo, en una relación estrecha entre lo que es el modelo, lo que son las competencias, el actuar del docente en el desarrollo de este método y la forma que los estudiantes sean capaces de crear sus propios procedimientos para acceder al conocimiento, compartiendo con esto la responsabilidad del proceso

No es tanta la responsabilidad del profesor, como la responsabilidad del alumno, ¿por qué?, el alumno tiene que aprender a aprender, tiene que aprender a saber, tiene que aprender a ser, tiene que aprender a convivir, y eso se dice fácil, y eso es sola- mente la realidad ¿sí? Para que un alumno pueda tener éxito, no necesariamente requiere de un profesor, lo más importante en un alumno es, el interés personal de superación. Si el alumno quiere superarse lo hace con un profesor o pesar de un profesor, si el alumno no quiere por más técnicas pedagógicas que puedas utilizar no se va a lograr absolutamente nada (D3)

En esta lógica de interpretación docente encontramos una realidad que no es vigente en el modelo educativo, misma que nos habla del proceso de implementación como un todo en una parte de su ejecución, ya que como mencionamos anteriormente, el docente debe de contar con ciertas competencias a transmitir que el alumno debe de recibir. La mayoría de las veces, los alumnos no están formados bajo un esquema de aprendizaje que los haya envuelto en una dinámica didáctica de aprendizaje, pues cada profesor bajo su entendimiento desempeña una función distinta con métodos y técnicas diferentes. Por tanto, no se propicia la creación del espacio cooperativo en el que tanto alumno, como docente, lleven a cabo la reflexión correspondiente de su práctica.

\section{Conclusiones y recomendaciones}

De todas las entrevistas y las categorías surgieron los retos, estrategias y planes de propuesta que pueden servir como base para una mejor aplicación y comprensión del modelo. La estructura curricular que se generó es una fuente de referencia importante para que se den los cambios necesarios en la generación de nuevos recursos. Sin embargo, hay muchos retos surgieron con los resultados de las entrevistas y que empatan con las categorías de análisis. en el siguiente cuadro presentamos estos retos con sus respectivas propuestas y estrategias de aplicación.

Las categorías obtenidas en el transcurso de

Pensamiento Americano Vol. 11 - No. 21 · Julio-Diciembre • Corporación Universitaria Americana • Barranquilla, Colombia · ISSN: 2027-2448 . 
Tabla 1:

Propuestas, retos y estrategias

\begin{tabular}{|c|c|c|}
\hline Retos & Propuesta & Estrategla \\
\hline $\begin{array}{l}\text { Conocer y comprender } \\
\text { el modelo }\end{array}$ & $\begin{array}{l}\text {-Presentar el modelo de manera concisa, } \\
\text { precisa y clara los directivos y docentes, es- } \\
\text { pecificando los objetivos, estrategias y metas } \\
\text { que se pretende alcanzar con el modelo }\end{array}$ & $\begin{array}{l}\text { - Realización de seminarios } \\
\text { para explicarles a los docentes } \\
\text { el modelo y sus implicaciones }\end{array}$ \\
\hline $\begin{array}{l}\text { Llevar a la práctica el } \\
\text { modelo }\end{array}$ & $\begin{array}{l}\text {-Establecer un vínculo entre lo que se } \\
\text { busca en el modelo y la experiencia } \\
\text { académica y profesional que se tenga }\end{array}$ & $\begin{array}{l}\text { - Realización de autoevaluación } \\
\text { con un análisis FODA }\end{array}$ \\
\hline $\begin{array}{l}\text { Más habilidades de } \\
\text { investigación y elabora- } \\
\text { ción de contenidos }\end{array}$ & $\begin{array}{l}\text {-Colaborar en academias para poder crear } \\
\text { vínculos entre las diversas propuestas que } \\
\text { se tengan para el desarrollo de proyectos } \\
\text { y contenidos de aprendizaje }\end{array}$ & $\begin{array}{l}\text { - Juntas de academia y de } \\
\text { pares para establecer una } \\
\text { dinámica integral de lo que se } \\
\text { busca lograr }\end{array}$ \\
\hline $\begin{array}{l}\text { Cambio de mentalidad } \\
\text { y resistencia al cambio }\end{array}$ & $\begin{array}{l}\text { - Los docentes deben sentirse identifi- } \\
\text { cados con el proceso y con la institución }\end{array}$ & $\begin{array}{l}\text { - Elaboración de dinámicas } \\
\text { grupales de reflexión en las } \\
\text { academias con los docenes para } \\
\text { crear un vinculo de identificación }\end{array}$ \\
\hline $\begin{array}{l}\text { Estrategias de compren- } \\
\text { sión y transmisión del } \\
\text { conocimiento }\end{array}$ & $\begin{array}{l}\text {-Desarrollar habilidades que necesita el } \\
\text { docente para transmitir el conocimien- } \\
\text { to desde su experiencia }\end{array}$ & $\begin{array}{l}\text { - Realización de cursos sobre } \\
\text { diversos recursos didácticos que } \\
\text { puedan aplicar en clase partir } \\
\text { de un enfoque de la formación y } \\
\text { desarrollo de competencias }\end{array}$ \\
\hline $\begin{array}{l}\text { Atender al alumno } \\
\text { desde una perspectiva } \\
\text { integral }\end{array}$ & $\begin{array}{l}\text {-Desarrollar en los alumnos la inquietud } \\
\text { de conocer, de aprender y del pensa- } \\
\text { miento critico-reflexivo, proporcionán- } \\
\text { dole recursos que pueda aprovechar }\end{array}$ & $\begin{array}{l}\text { - Establecimiento de una relación } \\
\text { de comprensión yentendimiento } \\
\text { con los alumnos para ser partíipes } \\
\text { directos de su formación profesional } \\
\text { yacadémica }\end{array}$ \\
\hline Liderazgo & $\begin{array}{l}\text {-Desarrollar en los docentes un sentido } \\
\text { de liderazgo transformacional acorde a } \\
\text { lo que se necesita en la implementa- } \\
\text { ción del modelo }\end{array}$ & $\begin{array}{l}\text { - Talleres de desarrollo de poten- } \\
\text { ciales para fomentar un tipo de } \\
\text { liderazgo transformacional }\end{array}$ \\
\hline
\end{tabular}

la investigación dejan retos que los docentes consideran como básicos, esenciales y fundamentales para determinar la viabilidad del modelo por competencias. Entre ellos surgió la necesidad de hacerlos participes en los espacios de interacción de que surjan en la toma de decisiones puesto que, al sentirse fuera de contexto, se crean conflicto en la asimilación de la información. Lo anterior se refleja en que perciben que el modelo es interesante pero no tiene la suficiente fuerza puesto que no ha existido una indicción sobre la practica del mismo.

También establecen el reto de hacer mas integral la educación de los alumnos mediante estrategias bien definidas que el mismo modelo establece, pero que ellos, si no es por iniciativa propia, desconocen. Lo anterior de genera como una parte en que el trabajo en academia debe establecer prioridades y donde el liderazgo por parte de los directivos, jefes de departamento y de academia deben considerar como parte fundamental para el trabajo en conjunto. Como lo definieron, en ocasiones se abandona el trabajo colegiado y sólo tienen que acatar indicaciones sobre lo que debe implementar en el aula, sin saber la finalidad del mismo.

Durante esta investigación pudimos cerciorarnos que existen muchas incógnitas cuando se quiere generar un cambio en las organizaciones. Una de las partes más importantes dentro de las universidades es la implementación de modelosy las reformas curriculares que buscan cambios exponenciales. Desde la mirada de los actores, con la aplicación del MSXXI su campo de acción experimentó cambios importantes. Sin embargo, no es tan probable que dichos cambios vayan encaminados a cumplir con los objetivos que establece el modelo, sino más bien son cambios que ellos mismos han propiciado y adoptado con la finalidad de disminuir o evitar la incertidumbre. También encontramos que los docentes del ITMMZ tienen una concepción distinta en cuanto al proceso de aprendizaje y a propiciar la construcción de conocimiento en los alumnos, y que éste se involucre y sea participativo tal y como lo establece el modelo. La parte de los planes y programas no puede ser ejecutada si no existe una guía que empareje todos los procesos para que los docentes lo comprendan, lo ejecuten y lo evalúen. Si esto se realiza así, pueden surgir una serie de alternativas para generar conocimiento aplicable en los alumnos (competencias). Sin embargo, la forma de realizarlo, lo que es necesario para lograrlo y lo que se debe de saber antes de hacerlo, no es conocido por los ejecutantes.

Según lo establecen las posturas teóricas respecto a esto, un modelo debe de contener diferentes puntos que creen una adecuada vinculación entre los interesados. Además de ello, es necesario crear una cultura que acerque a los mismos actores al desarrollo de una concep- 
ción sobre el proceso de enseñanza-aprendizaje que sea significativo para los alumnos que además sirva como eslabón en la concepción de un modelo integral Es por ello que el modelo debe de tomar una visión general de las problemáticas y adaptarla al contexto mediante el cual se genera el uso de los recursos con los que s formularse, implementarse y evaluarse. En el caso de la educación superior tecnológica de México, aún no es claro el rumbo que tomará o si seguirá formando parte del esquema mediante el cual de presta este tipo de educación. Lo cierto es que aún hay que establecer una adecuada implementación del mismo mediante la concientización e interiorización por parte de los docentes para que pueda generar un resultado más cercano a lo esperado.

La evaluación que se obtuvo como resultado de la investigación nos dice que el modelo está ausente de un proceso claro de implementación por parte de los docentes lo cual impedimenta conocerlo y adaptarlo a la realidad en el aula. Por tal motivo, los procesos que los docentes llevan para implementar el modelo generar competencias no son los adecuados, por lo menos para el presente caso. Lo que nos viene a establecer que las ventanas y las puertas tienen funciones diferentes y cada individuo en su contexto les da uso. Las ventanas permiten visualizar un futuro y las puertas permiten entrar o salir de un contexto determinado. Las competencias y el MSXXI sirven como guía para realizar esto, y los docentes toman una perspectiva distinta en dependencia de si prefieren o les conviene más las puertas o las ventanas.

La educación superior tecnológica en México en su conjunto es la que atiende al mayor número de estudiantes por lo que tiene una responsabilidad muy importante al momento de ejecutar este tipo de cambios. Para ello es importante que se desarrollen investigaciones que tengan un campo de acción más amplio y que puedan desentrañar todas las problemá- ticas que se generan. Este trabajo solo abarcó un micro mundo de análisis y tuvo un enfoque descriptivo que deja varias líneas de investigación para posteriores trabajos que evalúen el modelo y los resultados que ha tenido. Nosotros nos concentramos sólo en esta parte docente como un inicio de un trabajo que espera conocer las repercusiones del cambio curricular mediante este modelo educativo en la educación superior.

Las propuestas que surgen de ello inducen a establecer líneas específicas respecto a la viabilidad del modelo por competencias puesto que no existen indicadores definidos con los cuales poder profundizar el análisis. Los parámetros de medición con lo que se establezcan las competencias alcanzadas por parte de los alumnos, puede establecer las líneas a seguir en el desarrollo de una investigación más integral, pues en este caso, sólo se obtuvo la percepción de los docentes. Una percepción de los alumnos y directivos nos pude dar una visión más global sobre la implementación del modelo al igual que puede ser un punto de partida para desarrollar la propuesta de mejora o adecuación.

Uno de los puntos clave en el trabajo fue que lo docentes no se sienten parte del cambio y ello los orilla a concentrar su ánimo en otras actividades, o simplemente se aíslan y segmentan en grupos. Es por eso que se toma la metáfora de la isla de los olvidados como aquella donde los barcos encallan y los náufragos deben adaptarse a la nueva forma de vida. Por tanto, los olvidados son aquellos cuya voz y decisiones son relegadas a la conveniencia de otros. Para continuar con el análisis debemos de tomar en consideración a todos los actores que tienen incidencia en los procesos y no sólo a los que toman a las decisiones, los que formulan las propuestas o aquellos que evalúan a los que las ejecutan. 


\section{Referencias}

Cordero, O., Contreras, F., Fernandez, J., \& Hernández, A. (2016). La formación por competencias en la educación superior: alcances y limitaciones desde referentes de México, España y Chile. México: TH ebook.

Díaz-Barriga, F. R. (1997). Estrategias docentes para un aprendizaje significativo. México: Mc Graw-Hill.

Díaz Barriga, F. y Hernández, G. (2002). Estrategias docentes para un aprendizaje significativo. Una interpretación constructivista ( $2^{a}$. ed.). México: McGraw Hill.

Escudero Muñoz, J. M. (1981). Modelos didácticos: planificación sistemática y autogestión educativa. España: Oikos.

Escudero, E., \& Martínez, V. (2017). Adopción del modelo educativo por competencias, desde la categoría de campus. Actualidades de la investigación en educaión, 1-22.

García-Valcácer, A. (2002). Tecnologia educativa: CAracteristicas y evolución de una disciplina. Revista Educación y pedagogia. 14(33). Universidad de Salamanca.

García Fraile, A., Tobón, S., \& López Rodríguez, N. (2008). Los proyectos formativos: una estrategias didácticas para la formación por competencias. Lima: A.B.

García, J. (2011). Modelo educativo basado en competencias: importancia y necesidad. Actualidades investigativas en educación, 11(3), $1-24$.

García, M., Junyent, M., \& Fonolleda, M. (2017). How to assess professional competencies in Education for Sustainability?: An approach from a perspective of complexity. International Journal of Sustainability in Higher Education, 18(5), 772-797.

Garzón, C. (2016). Implicaciones curriculares del enfoque por competencias en educación superior. Boletín virtual, 1-25.

Gonczi, A., \& Athanasou, J. (1996). Intrumentación de la educación basada en competencias. Perspectiva de la teoría y la práctica en Australia. México: Limusa.
González, G. I. (2014). La implementación de un modelo educativo tecnológico desde la interpretación docente. El caso de las políticas del Instituto Tecnológico Superior de Zapopan. Tesis de maestría. Jalisco, México.

ITSZapopan. (2016). Sitio oficial. Obtenido de http:// www.itszapopan.edu.mx/

Lasnier, F. (2000). La formación por competencias. Madrid: Guerin.

Ortega, F. (2017). Principios e implicaciones del nuevo modelo educativo. RIIEE, XLVII(1), 43-62.

Senge, P. (2002). Escuelas que aprenden. Un manual de la Quinta disciplna para educadores, padres de familia y todos lo que se interesen en la educación. (Norma, Ed.) 17-71.

SEP. (2013). Sistema Nacional de Informaciòn Educativa. Obtenido de http://www.snie.sep.gob. $\mathrm{mx}$ /indicadores_x_entidad_federativa.html

SES. (2013). Portal principal DGEST. http://www.snit. $\mathrm{mx}$ /informacion/sistema-nacional-de-educacionsuperior-tecnologica.

SNEST. (2013). Modelo Educativo para el Siglo XXI. México: SEP.

SNIT. (2003). Modelo educativo para el tercer milenio. México: SEP.

Stan, J. (2017). Competence" and occupational standards: observations from six European countries". Education+Training, 59(2), 23-39.

Stan, L., Koniotaki, A., \& Religa, J. (2018). ComProCom: a revised model of occupational competence. Emerald Publishing, 40(4), 290-302.

TecNM. (2015). Manual de operaciones AcadémicoAdministrativo del Tecnológico Nacional de México. México: SEP.

TecNM. (2017). Pagina principal TecNM. Obtenido de http://www.tecnm.mx/informacion/sistemanacional-de-educacion-superior-tecnologica

Villagrán, S., Jasso, D., Aldaba, M. \& Rodríguez, M. (2017). Mobbing: impacto psicológico en docentes universitarios, repercusiones en el sentido de pertenencia y permanencia laboral. Pensamiento Americano, 10(18), 77-95. http:// dx.doi.org/10.21803\%2Fpenamer.10.18.388 
Wildman, P. (2003). Al borde del conocimiento: hacia las multiuniversidades polifónicas. En S. Inayatullah, J. Gidley, \& Pomares (Ed.), La universidad en transformación. Perspectivas globales sobre los futuros de la universidad (págs. 131-139). Barcelona: Pomares.

Zapata, J. (2015). El modeloy enfoque por competencias en la educación superio: apuntes sobre sus fortalezas y debilidades. Revista académia y virtualidad, 8(2), 24-33. 